\title{
The Validity and Sensitivity of PANSS-6 in the Clinical Antipsychotic Trials of Intervention Effectiveness (CATIE) Study
}

\author{
Søren D. Østergaard ${ }^{*, 1,2}$, Leslie Foldager ${ }^{3,4}$, Ole Mors ${ }^{1,2}$, Per Bech ${ }^{5}$, and Christoph U. Correll ${ }^{6-8}$ \\ ${ }^{1}$ Department of Clinical Medicine, Aarhus University, Aarhus, Denmark; ${ }^{2}$ Psychosis Research Unit, Aarhus University Hospital, \\ Risskov, Denmark; ${ }^{3}$ Department of Animal Science, Aarhus University, Tjele, Denmark; ${ }^{4}$ Bioinformatics Research Centre (BiRC), \\ Aarhus University, Aarhus, Denmark; ${ }^{5}$ Psychiatric Research Unit, Psychiatric Centre North Zealand, Copenhagen University Hospital, \\ Hillerød, Denmark; ${ }^{6}$ Division of Psychiatry Research, The Zucker Hillside Hospital, Glen Oaks, NY; ${ }^{7}$ Department of Psychiatry and \\ Molecular Medicine, Hofstra Northwell School of Medicine, Hempstead, NY; ${ }^{8}$ Department of Psychiatry and Behavioral Sciences, \\ Albert Einstein College of Medicine, Bronx, NY \\ *To whom correspondence should be addressed; Psychosis Research Unit, Aarhus University Hospital, Risskov, Skovagervej 2, 8240 \\ Risskov, Denmark; tel: + 45-61282753, fax: +45-78471609, e-mail: soeoes@rm.dk
}

It was recently demonstrated in acutely exacerbated schizophrenia that a 6-item version $($ PANSS-6: $\mathrm{P1}=$ delusions, $\mathbf{P} 2=$ conceptual disorganization, $\mathbf{P 3}=$ hallucinations, $\mathrm{N} 1$ = blunted affect, $\mathrm{N} 4$ = social withdrawal, N6 = lack of spontaneity/flow of conversation) of the 30-item Positive and Negative Syndrome Scale (PANSS-30) was scalable (all items provide unique information regarding syndrome severity) and able to separate the effect of antipsychotics from placebo. Here, we tested the validity and sensitivity of PANSS-6 in the Clinical Antipsychotic Trials of Intervention Effectiveness (CATIE) outpatient study. We examined (1) the scalability of PANSS-6 and PANSS30; (2) the correlation between PANSS-6 and PANSS-30 total scores; (3) whether PANSS-6 could identify symptom remission (Andreasen criteria); and (4) the effect of the 5 antipsychotics studied in CATIE Phase-1, using PANSS-6 and PANSS-30 total scores as outcomes. We found that for the $\mathbf{5 7 7}$ subjects with complete PANSS ratings at baseline, month 1, 3, and 6, PANSS-6 was scalable, whereas PANSS-30 was not. In the 1432 subjects in the intention-to-treat (ITT) sample, PANSS-6 and PANSS-30 total scores were highly correlated (Spearman correlation coefficient $=0.86$ ). Based on 5080 ITT ratings, PANSS-6 identified symptom remission with an accuracy of 0.99 $(95 \%$ confidence interval $=0.99-0.99)$. In ITT analyses, PANSS-6 and PANSS-30 identified the same statistically significant differences in antipsychotic efficacy, ie, olanzapine was superior to risperidone $(\boldsymbol{P}$-value PANSS-6 $=$ 0.0003 and PANSS-30 $=0.0003)$ and ziprasidone $(P$-value PANSS-6 $=0.0018$ and PANSS-30 $=0.0046)$. In conclusion, PANSS-6 is a brief schizophrenia rating scale that adequately measures severity, remission, and antipsychotic efficacy related to core positive and negative symptoms in clinical trials. Prospective studies of PANSS-6 in clinical practice are required.

Key words: schizophrenia/Psychiatric Status Rating Scales/psychometrics/psychopathology/antipsychotic agents

\section{Introduction}

The 30-item Positive and Negative Syndrome Scale (PANSS-30) ${ }^{1}$ is the most widely used measure of the severity of schizophrenia and related psychotic disorders in clinical studies. ${ }^{2}$ However, despite having been available for 30 years, the psychometric validity of PANSS-30 is still debated..$^{3-6}$

Recently, ${ }^{7}$ by means of item response theory (IRT) analysis, ${ }^{4}$ we evaluated the psychometric validity of the PANSS-30 and 2 suggested shorter versions of the PANSS, the 14-item version suggested by Levine et $\mathrm{al}^{3}$ and the 8 -item version suggested by Andreasen et $\mathrm{al}^{8}$ (the items considered in the definition of remission). None of these 3 scales were scalable (scalability is present when each item in a scale contains unique information regarding the severity of the latent syndrome being measured). The results suggested that for these scales adding the individual item scores to a total score is not meaningful from a psychometric perspective. However, only 2 items from the Andreasen et al criteria were responsible for its lack of scalability, namely G5 = mannerisms/posturing and G9 = unusual thought content. When conducting the Rasch IRT analysis on the remaining 6 items from the Andreasen et al criteria $(\mathrm{P} 1=$ delusions, $\mathrm{P} 2=$ conceptual disorganization, $\mathrm{P} 3=$ hallucinations, $\mathrm{N} 1=$ blunted affect, N4 = social withdrawal, N6 = lack of spontaneity/ 
flow of conversation), we found that they defined a scalable measure of "core" positive and negative symptoms of schizophrenia. Subsequently, we demonstrated that this "PANSS-6" scale was able to separate the effect of a typical antipsychotic (haloperidol) and an atypical antipsychotic (sertindole) from that of placebo in acutely exacerbated patients with schizophrenia. ${ }^{7}$ This finding opened the possibility of using the PANSS-6 as a psychometrically valid and more practical measure of the core psychopathology of schizophrenia in clinical trials. ${ }^{6}$

However, the initial PANSS-6 study described above was based on data from the randomized controlled trials by Zimbroff et $\mathrm{al}^{9}$ and van Kammen et $\mathrm{al}^{10}$ in acutely ill hospitalized patients with schizophrenia. Thus, currently, the validity of PANSS- 6 for patients in the chronic illness phase remains unknown. Therefore, we tested the psychometric properties of PANSS-6 further via a reanalysis of data from the Clinical Antipsychotic Trials of Intervention Effectiveness (CATIE) study, which represents the largest randomized clinical effectiveness trial in chronic schizophrenia. ${ }^{11}$ Specifically, the following 4 research questions were addressed:

1. Are PANSS-6 and PANSS-30 scalable measures of the severity of schizophrenia in CATIE?

2. How well do PANSS-6 total scores correlate with PANSS-30 total scores-ie, does PANSS-6 convey similar information as PANSS-30?

3. Can PANSS-6 capture "cross-sectional" remission as defined by the Andreasen et $\mathrm{al}^{8}$ criteria?

4. Is PANSS-6 equally sensitive as PANSS-30 in detecting differences in antipsychotic efficacy?

\section{Methods}

\section{CATIE Data}

We used the phase 1 data from CATIE, conducted between January 2001 and December 2004, which is described in detail elsewhere. ${ }^{11-13}$ In brief, 1493 individuals with chronic but nonrefractory schizophrenia, aged 18-65 and meeting (or having met) the Diagnostic and Statistical Manual of Mental Disorders, Fourth Edition (DSM-IV) criteria $^{14}$ for schizophrenia were recruited at 57 sites across the United States. Individuals with first-episode or treatment-resistant schizophrenia were excluded. Participants were randomized to the first-generation antipsychotic perphenazine, or one of the following second-generation antipsychotics, olanzapine, risperidone, quetiapine, or ziprasidone. Ziprasidone was approved for use by the Food and Drug Administration (FDA) after trial initiation and was added to the study in January 2002. Participants remained on the assigned treatment for up to 18 months or until discontinuation (phase 1 of CATIE). Although CATIE had several phases, ${ }^{11-13}$ for the current study, we analyzed the PANSS-30 ratings from CATIE phase $1 .{ }^{11}$

\section{Statistical Analysis}

The statistical methods used for each of the 4 research questions addressed in this study are outlined below:

Scalability of PANSS-6 and PANSS-30. Following the approach from our prior test of the scalability of PANSS- 6 and PANSS-30, ${ }^{7}$ the Rasch rating scale model was tested by means of the RUMM2030 Program. ${ }^{15}$ As described in further detail elsewhere, ${ }^{7}$ the null hypothesis of scalability in this model is that there is an invariant ordering of items, such that some have high prevalence (covering milder symptoms), while others have progressively lower prevalence (covering progressively more severe symptoms). This hypothesis was tested with $\chi^{2}$ statistics that are robust indicators of the item fit for the Rasch analysis. ${ }^{16}$ If the $\chi^{2}$ value is low and the $P$ value is $>.01$ (it is customary to use the 0.01 level for statistical significance in these analyses ${ }^{4}$ ), then the null hypothesis is accepted and the scale has demonstrated scalability. ${ }^{4}$ In the present study, the scalability of PANSS-6 and PANSS-30 was evaluated by means of the $\chi^{2}$ test at baseline, month 1, 3, and 6 to test for scalability over time. For this analysis, in agreement with our recent study of scalability of PANSS-6 and PANSS-30 in acutely exacerbated inpatients with schizophrenia, ${ }^{7}$ we only included participants with complete PANSS ratings at baseline, and month 1, 3, and 6. Six months was chosen as the endpoint for the scalability analysis, as an acceptable number of participants still remained active in CATIE at that time. ${ }^{11}$ However, to test whether or not the resultant findings were driven by the 6-month completer analysis, we conducted a sensitivity analysis, repeating the primary Rasch analysis based on the baseline PANSS ratings of the "dropouts" (those without complete ratings for 6 months), assessing the consistency with the results of the primary analysis. For scales proving to be scalable in the initial analysis described above, we used the differential item functioning (DIF) analysis in RUMM203015 to examine whether age, gender, time (baseline, month 1,3 , and 6), or treatment allocation influenced the rank order of the items. Again, $P$ values $>.01$ indicated that the Rasch model for DIF was accepted.

\section{Correlation Between PANSS-6 and PANSS-30 Total} Scores. The correlation between PANSS-6 total scores and PANSS-30 total scores from the entire CATIE study (ratings $=5081)$ was performed by means of Spearman correlation analysis. For PANSS-6 and PANSS-30, we also assessed the correlation between the relative change in total score [(current total score - baseline score) / baseline score], the total score ratio to baseline (current total score / baseline score), as well as the total score $\log$ (ratio to baseline) [ie, $\log$ (current total score / baseline total score), which corresponds to: $\log$ (current total score $)-\log ($ baseline total score $)]$. These 3 correlations 
were based on 3929 ratings, ie, 5081 ratings minus the baseline ratings.

Adequate Capturing of (Cross-Sectional) Remission Defined by the Andreasen et al Criteria by PANSS-6. The "Andreasen et al criteria" 8 are often used to define symptom remission in schizophrenia, which is reached when a patient scores $\leq 3$ (="mild") on the following 8 PANSS items: $\mathrm{P} 1=$ delusions, $\mathrm{P} 2=$ conceptual disorganization, $\mathrm{P} 3=$ hallucinations, $\mathrm{N} 1=$ blunted affect, $\mathrm{N} 4=$ social withdrawal, N6 = lack of spontaneity/flow of conversation, G5 = mannerisms/posturing, and G9 = unusual thought content. Since PANSS- 6 consists of 6 of these 8 items, we tested whether PANSS- 6 could accurately identify symptom remission in schizophrenia, using the Andreasen et al criteria ${ }^{8}$ as reference/index of validity. In the Andreasen et al criteria, maintenance of simultaneous ratings of $\leq 3$ (mild) over a 6-month period is required for symptom remission. However, since a very large proportion of the participants in CATIE phase 1 had dropped out prior to 6 months, we focused on "cross-sectional" remission, ie, the agreement between meeting PANSS-6 symptom remission criteria (score of $\leq 3$ on each of the 6
PANSS-6 items) and meeting the Andreasen et al symptom remission criteria (score of $\leq 3$ on the 8 items mentioned above) at any single rating occasion throughout the CATIE Phase $1(n=5080)$.

PANSS-6 Sensitivity to Differences in Antipsychotic Efficacy Using PANSS-30 as the Reference. To test whether PANSS-6 could identify the same differences as PANSS-30 in the efficacy of the antipsychotics used in CATIE phase 1, we used linear mixed-effects models with subject random intercepts and with drug specific means determined by third order polynomials in time. Within-subject serial correlation between measures at different follow-up days was handled by a continuous time autoregressive process of order one. The actual follow-up day (visit day after baseline) measured in decimal weeks was used for these analyses with $\log$ (ratio to baseline) [ie, $\log$ (current total score / baseline total score)] as the outcome. The log(ratio to baseline) was chosen as the outcome based on the observation evident from the correlations shown in figure 1, namely that the logarithmic transformation reduced the increasing spread of PANSS- 6 ratios with increasing PANSS-30 ratios (compare the bottom
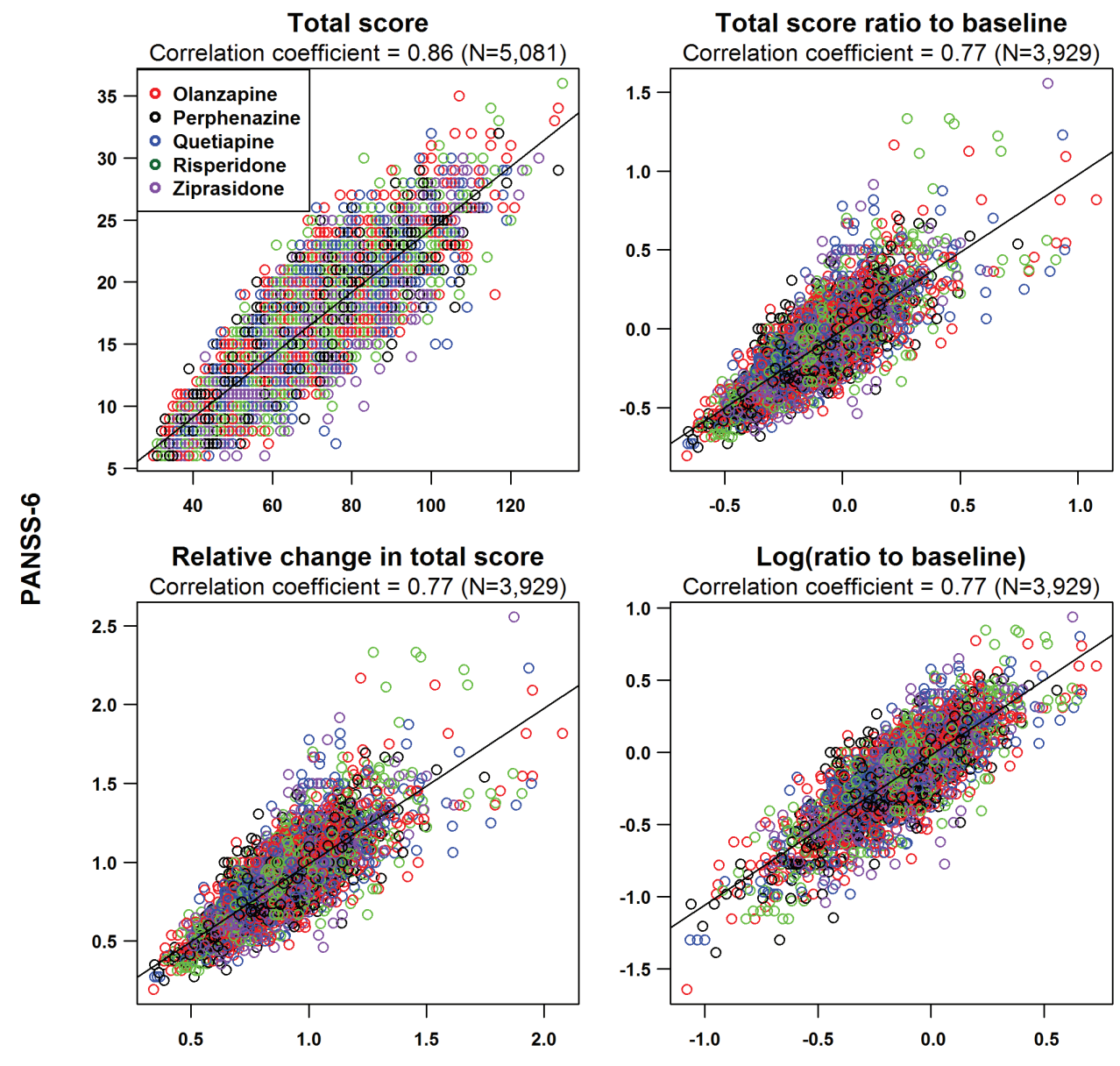

PANSS-30

Fig. 1. Correlation between PANSS-6 and PANSS-30 total scores. 
right correlation to the top right correlation in figure 1). Use of the $\log$ (ratio to baseline) resulted in stable variability between PANSS-6 and PANSS-30 throughout the range and thus a more reasonable comparison of the two. Moreover, residuals were in much better agreement with the normal distribution assumption of the mixedeffects model when the $\log$ (ratio to baseline) was used, whereas residuals from the ratios had a tendency of right skewness.

For meaningful interpretation of the polynomial intercept parameters, we subtracted 4 weeks from the decimal weeks and used this result as the time variable - the rationale being that first follow-up was carried out at around week 4 and thus that the intercept measures immediate treatment effect. A third order polynomial was chosen to allow for a reasonably flexible evolvement that could be used for all drugs (a polynomial of lower order could be used for some drugs, whereas a higher order was found not to give a better fit in any case). Comparison between pairs of drugs was carried out by the 4 degrees of freedom likelihood ratio test of the null hypothesis that all 2 times 4 coefficients in the polynomial were equal. Consistent with the study by Lieberman et al, ${ }^{11}$ slightly different datasets were used for the pairwise comparisons: SET 1: Total sample excluding those with tardive dyskinesia (not eligible for perphenazine) and those receiving ziprasidone (late entry in the trial). This set was used for the comparison of perphenazine with olanzapine, quetiapine and risperidone. SET 2: Total sample including those with tardive dyskinesia and excluding those receiving ziprasidone (late entry in the trial) and perphenazine. This set was used for the pairwise comparisons between olanzapine, quetiapine, and risperidone. SET 3: Participants enrolled after ziprasidone was included - including those with tardive dyskinesia and excluding those receiving perphenazine. This set was used for the comparisons of ziprasidone with olanzapine, quetiapine, and risperidone. SET 4: Participants enrolled after ziprasidone was included - excluding those with tardive dyskinesia. This set was used for the comparison between ziprasidone and perphenazine. The likelihood ratio tests comparing the antipsychotics were adjusted for exacerbation (SET 1-4) and tardive dyskinesia (SET 2-3). As there were 10 pairwise comparisons of antipsychotics, the Bonferroni-adjusted threshold for statistical significance was $0.05 / 10=0.005$ to maintain a significance level of 0.05 .

\section{Results}

The results obtained for each of the 4 research questions addressed in this study are outlined below:

\section{Scalability of PANSS-6 and PANSS-30}

The results of the Rasch rating scale model for PANSS-6 and PANSS-30 are shown in table 1A. While PANSS-6 was accepted as being scalable at all 4 time points considered in the analysis, PANSS-30 was rejected at all 4 time points. The Rasch locations for the PANSS- 6 items at baseline, month 1,3, and 6 are shown in table $1 \mathrm{~B}$.

The mean Rasch-locations over the 4 time points (rightmost column in table 1B) indicate that the prevalence-severity hierarchy of the PANSS-6 items for the

Table 1. Results of the Rasch Analyses

A.

\begin{tabular}{|c|c|c|c|c|}
\hline & Baseline & Month 1 & Month 3 & Month 6 \\
\hline PANSS-6 & $\begin{array}{l}\text { Accepted } \\
\chi^{2}=59.71 \\
P=0.120\end{array}$ & $\begin{array}{l}\text { Accepted } \\
\chi^{2}=68.42 \\
P=0.028\end{array}$ & $\begin{array}{l}\text { Accepted } \\
\chi^{2}=68.20 \\
P=0.029\end{array}$ & $\begin{array}{l}\text { Accepted } \\
\chi^{2}=53.50 \\
P=0.271\end{array}$ \\
\hline PANSS-30 & $\begin{array}{l}\text { Rejected } \\
\chi^{2}=563.77 \\
P<0.001\end{array}$ & $\begin{array}{l}\text { Rejected } \\
\chi^{2}=448.60 \\
P<0.001\end{array}$ & $\begin{array}{l}\text { Rejected } \\
\chi^{2}=475.02 \\
P<0.001\end{array}$ & $\begin{array}{l}\text { Rejected } \\
\chi^{2}=513.72 \\
P<0.001\end{array}$ \\
\hline
\end{tabular}

B.

\begin{tabular}{|c|c|c|c|c|c|}
\hline Item & Baseline & Month 1 & Month 3 & Month 6 & Mean \\
\hline Delusions (P1) & $-0.200[1]$ & $-0.064[2]$ & $-0.018[3]$ & $0.068[5]$ & $-0.054[3]$ \\
\hline Conceptual disorganization (P2) & $0.006[4]$ & $0.047[5]$ & $0.110[6]$ & $0.046[3]$ & $0.052[4]$ \\
\hline Hallucinations (P3) & $0.267[6]$ & $0.280[6]$ & $0.106[5]$ & $0.151[6]$ & $0.201[6]$ \\
\hline Blunted affect (N1) & $-0.074[3]$ & $-0.011[3]$ & $-0.121[2]$ & $-0.076[2]$ & $-0.071[2]$ \\
\hline Social withdrawal (N4) & $-0.187[2]$ & $-0.265[1]$ & $-0.156[1]$ & $-0.238[1]$ & $-0.212[1]$ \\
\hline Lack of spontaneity and flow of conversation (N6) & $0.189[5]$ & 0.014 [4] & $0.079[4]$ & 0.048 [4] & 0.083 [5] \\
\hline
\end{tabular}

Note: A: Results of the item response theory analysis (Rasch) for PANSS- 6 and PANSS-30 at baseline, month 1, month 3 , and month 6 $(n=577) . P$ values for the $\chi^{2}$ test $>0.01$ are indicative of acceptable scalability. B: Rasch locations (ranks) for PANSS- 6 at baseline, month 1 , month 3 , and month 6 . The rank order is represented by the numbers in brackets. The numbers in the "Mean" column represent the mean of the locations at baseline, month 1 , month 3 , and month 6 . 
CATIE population is as follows: $\mathrm{N} 4=$ social withdrawal, $\mathrm{N} 1$ = blunted affect, $\mathrm{P} 1$ = delusions, $\mathrm{P} 2$ = conceptual disorganization, N6 = lack of spontaneity and flow of conversation, and $\mathrm{P} 3=$ hallucinations. This hierarchy means that while social withdrawal and blunted affect were associated with milder states of illness, lack of flow of conversation and hallucinations were indicative of more severe states of illness in the analyzed CATIE sample. ${ }^{4}$ The results of the DIF analysis showed that gender, age, treatment allocation, and time (baseline, month 1, month 3 , and month 6) did not significantly influence the rank order of the PANSS-6 items (supplementary table 1).

In order to ensure that the findings described above (PANSS-6 was scalable, while PANSS-30 was not) were not driven by the selection of the 6-month completers for the analysis, we repeated the primary Rasch analysis based on the baseline PANSS ratings of the "dropouts" (those without complete ratings for 6 months). This sensitivity analysis was based on PANSS ratings from 849 individuals (the ITT population of 1432 minus the 577 included in our primary analysis minus 6 individuals with incomplete PANSS-ratings at baseline). The results of the analysis of the dropouts confirmed those of our primary analysis, namely that PANSS-6 was scalable, whereas PANSS-30 was not. Furthermore, the Rasch locations at baseline (severity hierarchy) of the items were identical for the dropouts and the completers (supplementary table 2). These results indicate that our primary scalability analysis was not biased due to selection.

\section{Correlation Between PANSS-6 and PANSS-30 Total Scores}

Results of the 4 correlation analyses described in the Methods section are outlined in figure 1 . The correlation between PANSS-6 and PANSS-30 total scores was high (Spearman correlation coefficient $=0.86$ ). Similarly, the correlation between PANSS-6 and PANSS-30 was high between the relative changes in total scores (Spearman correlation coefficient $=0.77$ ), between the total score ratios (Spearman correlation coefficient $=0.77$ ) and between the total score log-ratios (Spearman correlation coefficient $=0.77$ ).

\section{Adequate Capturing of (Cross-Sectional) Remission Defined by the Andreasen et al Criteria by PANSS-6}

There were 5080 PANSS ratings available for this analysis, 1464 (28.8\%) of which met the Andreasen et al criteria $^{8}$ for cross-sectional remission. PANSS- 6 captured these 1464 cases with a sensitivity of 1.00 (this result was foreseen a priori, as PANSS- 6 consists of 6 of the 8 items Andreasen et al criteria), a specificity of 0.99 (48 false positives), a positive predictive value of 0.97 , and a negative predictive value of 1.00 , which sums to an overall accuracy of 0.99 (95\% confidence interval
$[C I]=0.99-0.99)$. The $48(0.94 \%)$ of the 5080 PANSS ratings, representing false positive remission (ie, meeting PANSS-6 remission criteria, but not the Andreasen et al criteria), had a score of $4=$ moderate (and not of $5=$ moderate severe, $6=$ severe, or $7=$ extreme) on either item G5 = mannerisms/posturing $(n=18)$, item G9 = unusual thought content $(n=29)$ or both $(n=1)$. Figure 2 contains Kaplan-Meier plots of remission (the probability of nonremission) for each antipsychotic over the course of the CATIE study. The trajectories of the Kaplan-Meier curves for the individual antipsychotics (see color code) are almost identical for PANSS-6 and PANSS-30, ie, the similarity between remission as defined by PANSS- 6 and the Andreasen et al criteria is not biased by the individual antipsychotics studied in CATIE phase 1. SET 1, SET 2, SET 3, and SET 4 refers to those described in PANSS-6 sensitivity to differences in antipsychotic efficacy using PANSS-30 as the reference section under Methods section.

\section{Test of PANSS-6 Sensitivity to Differences in Antipsychotic Efficacy Using PANSS-30 as the Reference}

The results ( $P$ values) of the likelihood ratio tests comparing the efficacy of the 5 antipsychotics employed in the CATIE phase 1 study are provided in table 2 .

The results show that PANSS-6 and PANSS-30 identified the exact same statistically significant (Bonferroniadjusted level $=0.005$ ) differences in antipsychotic efficacy, namely that olanzapine was superior to risperidone $(P$-value PANSS- $6=0.0003$ and PANSS-30 $=$ $0.0003)$ and ziprasidone $(P$-value PANSS- $6=0.0018$ and PANSS-30 $=0.0046)$. This overlap of results obtained with PANSS-6 and PANSS-30 is further illustrated in figure 3, which shows the estimated polynomials of order 3 describing the trajectory of PANSS-30 and PANSS-6 scores with treatment time (weeks from baseline) as measured by the $\log$ (ratio).

\section{Discussion}

The aim of this study was to test the validity of PANSS$6^{7}$ in the CATIE phase 1 study, which represents the largest clinical trial with participants suffering from chronic schizophrenia. ${ }^{11}$ Specifically, 4 research questions were addressed, resulting in the following findings:

(1) Similar to our analyses in acutely exacerbated schizophrenia inpatients, ${ }^{7}$ in chronic outpatients

PANSS-6 was scalable, whereas PANSS-30 was not. Furthermore, the PANSS-6 item rank order was consistent over time, and across gender, age, and antipsychotic treatments. The ability of a rating scale to sustain the item rank order independently of these factors/characteristics is essential for its validity in comparing patients and treatments over time in a clinical trial or in clinical practice. $^{7,17}$ 


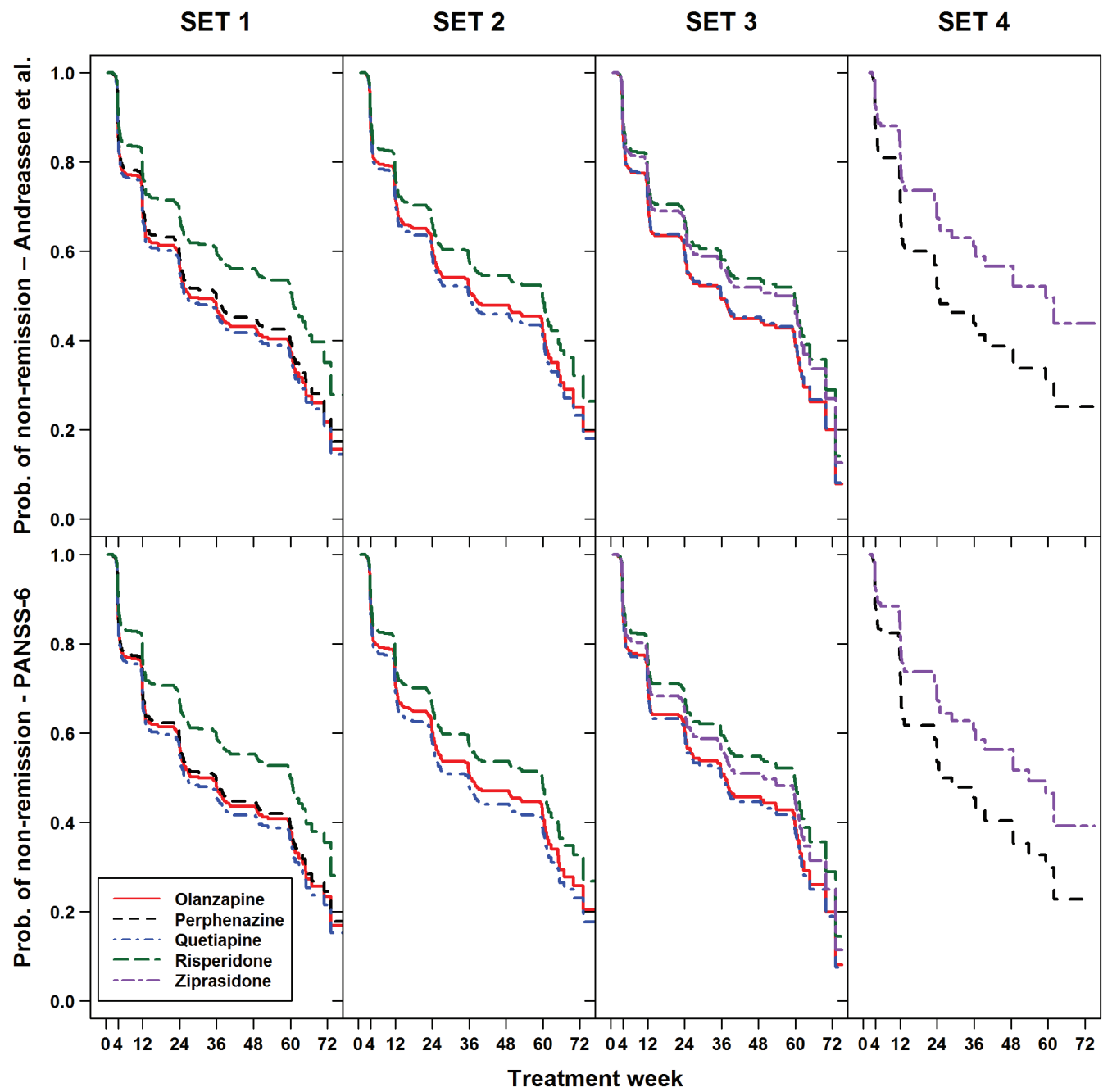

Fig. 2. Kaplan-Meier plots of non-remission as defined by the Andreasen et al criteria (top) and by PANSS-6 (bottom), stratified on the 5 antipsychotics studied in CATIE phase 1.

Table 2. $P$ Values of the Pairwise Likelihood Ratio Tests Comparing the 5 Antipsychotics in CATIE

\begin{tabular}{|c|c|c|c|c|}
\hline PANSS-30 & Perphanazine & Risperidone & Quetiapine & Olanzapine \\
\hline Risperidone & 0.24 & & & \\
\hline Quetiapine & 0.31 & 0.082 & & \\
\hline Olanzapine & 0.21 & $0.00031^{\mathrm{a}}$ & 0.030 & \\
\hline Ziprasidone & 0.016 & 0.0068 & 0.25 & $0.0046^{\mathrm{a}}$ \\
\hline PANSS-6 & Perphanazine & Risperidone & Quetiapine & Olanzapine \\
\hline Risperidone & 0.13 & & & \\
\hline Quetiapine & 0.80 & 0.15 & & \\
\hline Olanzapine & 0.59 & $0.00030^{\mathrm{a}}$ & 0.16 & \\
\hline Ziprasidone & 0.14 & 0.030 & 0.25 & $0.0018^{a}$ \\
\hline
\end{tabular}

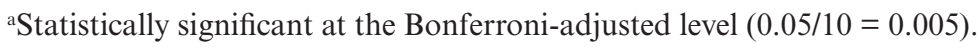

Interestingly, the Rasch locations (severity hierarchy) of the PANSS-6 items observed in this study of chronic outpatients $(\mathrm{N} 4=$ social withdrawal, $\mathrm{N} 1=$ blunted affect, $\mathrm{P} 1=$ delusions, $\mathrm{P} 2$ = conceptual disorganization, N6 = lack of spontaneity and flow of conversation, and P3 = hallucinations) were different from those found in our prior study of acutely exacerbated inpatients $(\mathrm{P} 1=$ delusions, $\mathrm{P} 3=$ hallucinations, $\mathrm{P} 2=$ conceptual disorganization, $\mathrm{N} 4=$ social withdrawal, $\mathrm{N} 1$ = blunted affect, N6 = lack of spontaneity and flow of conversation). ${ }^{7}$ In fact, the ranking was inverse, with lower severity (but higher prevalence) of the negative symptoms 


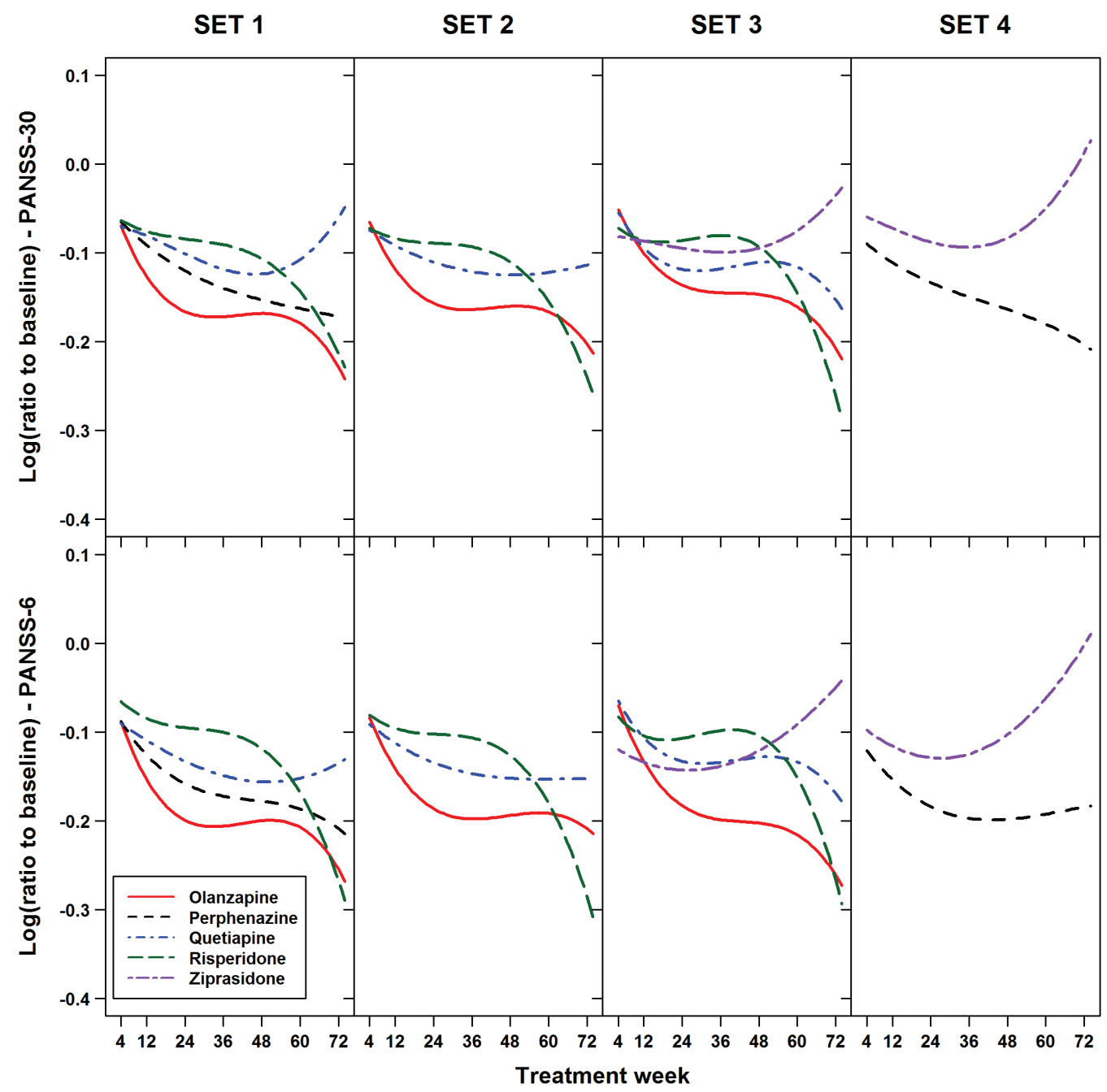

Fig. 3. The trajectory of PANSS-30 and PANSS-6 scores during CATIE Phase 1, stratified by treatment. Note: Estimated polynomials of order 3 describing trajectories of PANSS-30 and PANSS-6 scores with treatment (weeks from baseline) as measured by the log(ratio to baseline). The models included drug specific coefficients, 4 parameters for each drug, and were adjusted for tardive dyskinesia (SET 2 and SET 3) and exacerbation (all 4 sets). The curves shown are those estimated for individuals without tardive dyskinesia and exacerbation.

and higher severity (but lower prevalence) of the positive symptoms in the chronic outpatients, contrasting with lower severity (but higher prevalence) of the positive symptoms and higher severity (but lower prevalence) of the negative symptoms in acutely exacerbated patients. These findings likely reflect that the current study was based on PANSS ratings of patients with chronic schizophrenia in a relatively stable phase of illness, whereas our prior study was based on selectively higher PANSS ratings of acutely ill patients with exacerbated positive symptoms of schizophrenia. ${ }^{9,10}$ Curiously, however, lack of spontaneity and flow of conversation (N6) was of high severity and lower prevalence in both populations. Importantly, in both studies, DIF analysis demonstrated that the Rasch locations were stable over time, and across gender, age, and antipsychotic treatments.

(2) The total PANSS-6 and PANSS-30 scores were very highly correlated (Spearman correlation coefficient $=$ 0.86). Thus, it appears that although PANSS-6 contains
24 items less that PANSS-30, it essentially conveys the same core severity information for schizophrenia. Specifically, the squared Spearman correlation coefficient $(0.86 \times 0.86=0.74)$ indicates that approximately $74 \%$ of the variance in PANSS-30 is explained by PANSS-6. Put differently, given that PANSS- 6 covers 6 of the 8 symptoms included in the Diagnostic and Statistical Manual of Mental Disorders, Fifth Edition ${ }^{7,18}$ it seems that these specific $74 \%$ represent the core of schizophrenia covered by the much more extensive and laborious PANSS-30.

(3) At the cross-sectional level, PANSS-6 identified symptom remission as defined by the Andreasen et al criteria with very high accuracy $(0.99,95 \% \mathrm{CI}=0.99$ 0.99). This finding entails that using the psychometrically valid and even shorter PANSS-6 to define cross-sectional remission is equal in performance to the Andreasen et al criteria. Furthermore, since this analysis was based on 5080 ratings from the entire phase 1 of CATIE and only 48 cases $(<1 \%)$ of false positive classifications of 
remission on PANSS-6 compared to the Andreasen et al criteria were found, it seems likely that PANSS-6 can also validly classify remission longitudinally-for instance over 6 months as proposed by Andreasen et al. ${ }^{8}$ This possibility should be subjected to prospective testing.

(4) PANSS-6 and PANSS-30 identified the exact same statistically significant differences in antipsychotic efficacy in CATIE phase 1, namely that olanzapine was superior to both risperidone and ziprasidone. Thus, also in terms of detecting differences in the antipsychotic effect of drugs in chronic schizophrenia, PANSS-6 was as sensitive as PANSS-30. This finding is consistent with the results from our prior analysis of 2 studies $^{9,10}$ of acutely ill hospitalized patients with schizophrenia, where PANSS-6 was able to detect superior efficacy of haloperidol and sertindole compared to placebo. ${ }^{7}$

There are a number of limitations to consider when interpreting the results of this study. First, the CATIE study was not prospectively planned to enable psychometric validation of PANSS-6. However, a positive consequence of this limitation is that the PANSS ratings are unlikely to be biased in favor of PANSS-6 since PANSS-6 had not been established when the CATIE trial was conducted.

Second, since all participants in CATIE either met or had met DSM-IV criteria ${ }^{14}$ for schizophrenia, it is unclear whether the findings reported here are valid for other psychotic disorders and for schizophrenia operationalized by other diagnostic systems, most importantly for the DSM$5,{ }^{18}$ the International Classification of Disease, 10th revision (ICD-10) ${ }^{19}$ and the upcoming ICD-11. That being said, based on the similarity in the diagnostic criteria for schizophrenia in the DSM-IV, DSM-5, and ICD-10 (and those proposed for ICD-11), we have no reason to believe that the updates in the diagnostic manuals will have a large influence on the external validity of the results reported here. However, at least in section 3 of DSM$5,{ }^{18}$ dimensional ratings of schizophrenia symptoms are proposed for clinical use. The utility and psychometric properties of these dimensional assessments in clinical care need to be studied and should be compared to the utility of the PANSS-6, evaluating also whether the combined use of these instruments is feasible and whether it provides additional value.

Third, we did not investigate PANSS-6 symptom remission over 6 months, which is considered to be the standard duration criterion for remission. ${ }^{8}$ However, since PANSS-6 captured "cross-sectional" remission as defined by the Andreasen et al. criteria with an accuracy of 0.99 in a total of 5080 rating occasions across the first 6 months of CATIE data, we are optimistic that it will also be able to do so beyond 6 months. However, this question should be subjected to prospective testing.

Fourth, the core psychopathology measured by PANSS-6 is of course only one element of assessment. In many situations, it will be of relevance for both researchers and clinicians to address multiple additional dimensions, including psychopathology, social and interpersonal functioning, subjective wellbeing, quality of life, adverse effects, and physical health. If researchers or clinicians are interested in broader symptomatology, other versions of PANSS can be used, such as the 8 -item version defined by the Andreasen et al criteria, ${ }^{8}$ the 14-item version suggested by Levine et $\mathrm{al}^{3}$ (consisting of the 7 positive symptom items and the 7 negative symptom items), or the full PANSS-30. ${ }^{1}$ Also, for patients with severe schizophrenia-associated symptoms of depression or anxiety, PANSS-6 will not capture these symptoms. In such cases, it will be relevant to also administer rating scales specifically addressing these symptom domains, such as the Calgary Depression Rating Scale ${ }^{20}$ or the Hamilton Anxiety Rating Scale. ${ }^{21}$ However, when assessing the core symptom severity of schizophrenia, we believe that PANSS-6 is a good option from a clinical and psychometric perspective. $^{4}$

Finally, and perhaps most importantly, a major limitation of this and the other studies of PANSS- 6 is that the data were extracted from studies using the full PANSS$30 .^{9-11,22}$ Therefore, it remains unknown whether it is possible to obtain sufficient information for PANSS-6 rating without using the full PANSS-30 interview, which takes up to $60 \mathrm{~min}$ to administer in some cases and which also includes elicitation of symptoms that might help rating the PANSS-6 items. That the same information with similar psychometric and clinical validity can be obtained from a much shorter interview is of course a prerequisite for the utility of PANSS-6. We recently developed the Simplified Negative and Positive Symptoms Interview (SNAPSI), which can be used to collect information for rating selected items from various rating scales (including the 6 PANSS-6 items). SNAPSI takes approximately 15 min to administer and has performed well in our pilot tests. Therefore, we plan to subject it to thorough clinical and psychometric validation in research and clinical settings in different countries ${ }^{23}$ in the near future.

In summary, studies of PANSS-6 so far suggest that this brief scale may represent an advance in clinical trials methodology relating to antipsychotic efficacy. We also believe that PANSS-6 can orient clinicians to a measurement-based assessment of core psychopathology to evaluate treatment effects, success with core symptom remission, warning of impending relapse, etc., and we are planning to test these possibilities in prospective clinical studies. Indeed, it seems that PANSS-6 has the potential to become a useful tool for feasible measurement-based care approaches in schizophrenia. Measurement-based care has already demonstrated its value in major depressive disorder, ${ }^{24}{ }^{42}$ but has been tested in schizophrenia to a much lesser extent. ${ }^{33-35}$ That being said, the core psychopathology measured by PANSS- 6 is but one element of assessment, and clinicians or researchers may wish to address multiple dimensions of psychopathology, 
intra- and interpersonal functioning, adverse effects, etc., in order to assess a broader range of aspects that are relevant for the understanding and management of a given patient in clinical practice and research.

\section{Supplementary Material}

Supplementary data are available at Schizophrenia Bulletin online.

\section{Funding}

This work was supported by the Lundbeck Foundation (R165-2013-15320 to S.D.Ø.).

\section{Acknowledgments}

S.D.Ø., L.F., O.M., and P.B. declare no conflicts of interest. C.U.C. has been a consultant and/or advisor to or has received honoraria from: AbbVie, Acadia, Actavis, Actelion, Alexza; Alkermes, Bristol-Myers Squibb, Cephalon, Eli Lilly, Forum, Genentech, Gerson Lehrman Group, IntraCellular Therapies, Janssen/J\&J, LB Pharma, Lundbeck, Medavante, Medscape, Merck, Neurocrine, Otsuka, Pfizer, ProPhase, Reviva, Roche, Sunovion, Supernus, Takeda, Teva, and Vanda. He has provided expert testimony for Bristol-Myers Squibb, Janssen, and Otsuka. He served on a Data Safety Monitoring Board for Eli Lilly, Janssen, Lundbeck, Pfizer, Takeda, and Otsuka. He received grant support from Bristol-Myers Squibb, Otsuka, Lundbeck, and Takeda.

\section{References}

1. Kay SR, Fiszbein A, Opler LA. The positive and negative syndrome scale (PANSS) for schizophrenia. Schizophr Bull. 1987;13:261-276.

2. Leucht S. Measurements of response, remission, and recovery in schizophrenia and examples for their clinical application. J Clin Psychiatry. 2014;75(suppl 1):8-14.

3. Levine SZ, Rabinowitz J, Rizopoulos D. Recommendations to improve the positive and negative syndrome scale (PANSS) based on item response theory. Psychiatry Res. 2011;188:446-452.

4. Bech P. Clinical Psychometrics. Oxford, UK: Wiley-Blackwell; 2012.

5. Khan A, Lewis C, Lindenmayer JP. Use of non-parametric item response theory to develop a shortened version of the Positive and Negative Syndrome Scale (PANSS). BMC Psychiatry. 2011;11:178.

6. Fleischhacker WW. The usefulness of rating scales in patients with schizophrenia. Acta Psychiatr Scand. 2016;133:435.

7. Østergaard SD, Lemming OM, Mors O, Correll CU, Bech P. PANSS-6: a brief rating scale for the measurement of severity in schizophrenia. Acta Psychiatr Scand. 2016;133:436-444.

8. Andreasen NC, Carpenter WT Jr, Kane JM, Lasser RA, Marder SR, Weinberger DR. Remission in schizophrenia: proposed criteria and rationale for consensus. Am $J$ Psychiatry. 2005;162:441-449.

9. Zimbroff DL, Kane JM, Tamminga CA, et al. Controlled, dose-response study of sertindole and haloperidol in the treatment of schizophrenia. Sertindole Study Group. Am J Psychiatry. 1997;154:782-791.

10. van Kammen DP, McEvoy JP, Targum SD, Kardatzke D, Sebree TB. A randomized, controlled, dose-ranging trial of sertindole in patients with schizophrenia. Psychopharmacology (Berl). 1996;124:168-175.

11. Lieberman JA, Stroup TS, McEvoy JP, et al.; Clinical Antipsychotic Trials of Intervention Effectiveness (CATIE) Investigators. Effectiveness of antipsychotic drugs in patients with chronic schizophrenia. N Engl J Med. 2005;353:1209-1223.

12. Swartz MS, Perkins DO, Stroup TS, McEvoy JP, Nieri JM, Haak DC. Assessing clinical and functional outcomes in the Clinical Antipsychotic Trials of Intervention Effectiveness (CATIE) schizophrenia trial. Schizophr Bull. 2003;29:33-43.

13. Stroup TS, McEvoy JP, Swartz MS, et al. The National Institute of Mental Health Clinical Antipsychotic Trials of Intervention Effectiveness (CATIE) project: schizophrenia trial design and protocol development. Schizophr Bull. 2003;29:15-31.

14. American Psychiatric Association. Diagnostic and Statistical Manual of Mental Disorders. 4th ed. Washington, DC: American Psychiatric Association; 1994.

15. Andrich D, Sheridan BE, Luo G. RUMM2030. Version 5.1. Perth, Australia: RUMM Laboratory Pty Ltd; 2010.

16. Bech P. Rating scales for affective disorders: their validity and consistency. Acta Psychiatr Scand Suppl. 1981;295:1-101.

17. Allan ER, Sison CE, Alpert M, Connolly B, Crichton J. The relationship between negative symptoms of schizophrenia and extrapyramidal side effects with haloperidol and olanzapine. Psychopharmacol Bull. 1998;34:71-74.

18. American Psychiatric Association. Diagnostic and Statistical Manual of Mental Disorders. 5th ed. Washington, DC: American Psychiatric Association; 2013.

19. World Health Organization. The ICD-10 Classification of Mental and Behavioural Disorders. Diagnostic Criteria for Research. Geneva: WHO; 1993.

20. Addington D, Addington J, Maticka-Tyndale E. Assessing depression in schizophrenia: The Calgary depression scale. Br J Psychiatry Suppl. 1993:39-44.

21. Hamilton M. The assessment of anxiety states by rating. $\mathrm{Br} J$ Med Psychol. 1959;32:50-55.

22. Hochstrasser L, Borgwardt S, Lambert M, et al. Association of Positive and Negative Syndrome Scale short forms with global functioning and quality of life. Acta Psychiatr Scand. 2016;134:563-565.

23. Daniel DG, Alphs L, Cazorla P, Bartko JJ, Panagides J. Training for assessment of negative symptoms of schizophrenia across languages and cultures: comparison of the NSA-16 with the PANSS Negative Subscale and Negative Symptom factor. Clin Schizophr Relat Psychoses. 2011;5:87-94.

24. Trivedi MH, Rush AJ, Crismon ML, et al. Clinical results for patients with major depressive disorder in the Texas Medication Algorithm Project. Arch Gen Psychiatry. 2004;61:669-680.

25. Crismon ML, Trivedi M, Pigott TA, et al. The texas medication algorithm project: report of the texas consensus conference panel on medication treatment of major depressive disorder. J Clin Psychiatry. 1999;60:142-156. 


\section{S. D. Ostergaard et al}

26. Guo T, Xiang YT, Xiao L, et al. Measurement-based care versus standard care for major depression: a randomized controlled trial with blind raters. Am J Psychiatry. 2015;172:1004-1013.

27. Adli M, Berghöfer A, Linden M, et al. Effectiveness and feasibility of a standardized stepwise drug treatment regimen algorithm for inpatients with depressive disorders: results of a 2-year observational algorithm study. J Clin Psychiatry. 2002;63:782-790.

28. Adli M, Rush AJ, Möller HJ, Bauer M. Algorithms for optimizing the treatment of depression: making the right decision at the right time. Pharmacopsychiatry. 2003;36(suppl 3):S222-S229.

29. Trivedi MH, Rush AJ, Wisniewski SR, et al.; STAR*D Study Team. Evaluation of outcomes with citalopram for depression using measurement-based care in STAR *D: implications for clinical practice. Am J Psychiatry. 2006;163:28-40.

30. Adli M, Bauer M, Rush AJ. Algorithms and collaborativecare systems for depression: are they effective and why? A systematic review. Biol Psychiatry. 2006;59:1029-1038.
31. Ricken R, Wiethoff K, Reinhold T, et al. Algorithm-guided treatment of depression reduces treatment costs-results from the randomized controlled German Algorithm Project (GAPII). J Affect Disord. 2011;134:249-256.

32. Bauer M, Pfennig A, Linden M, Smolka MN, Neu P, Adli M. Efficacy of an algorithm-guided treatment compared with treatment as usual: a randomized, controlled study of inpatients with depression. J Clin Psychopharmacol. 2009;29:327-333.

33. Kane JM, Robinson DG, Schooler NR, et al. Comprehensive versus usual community care for first-episode psychosis: 2-year outcomes from the NIMH RAISE Early Treatment Program. Am J Psychiatry. 2016;173:362-372.

34. Miller AL, Crismon ML, Rush AJ, et al. The Texas medication algorithm project: clinical results for schizophrenia. Schizophr Bull. 2004;30:627-647.

35. Moore TA, Buchanan RW, Buckley PF, et al. The Texas Medication Algorithm Project antipsychotic algorithm for schizophrenia: 2006 update. J Clin Psychiatry. 2007;68:1751-1762. 